\title{
EDITORIAL
}

\section{A special issue on Biophotonics in Europe}

\author{
Valery V. TUCHIN ${ }^{1,2,3}$, Ekaterina BORISOVA ${ }^{4}$, Malgorzata JĘDRZEJEWSKA-SZCZERSKA ${ }^{5}$, \\ Martin J. LEAHY ${ }^{6}$, Francesco S. PAVONE ${ }^{7}$, Jürgen POPP ${ }^{8}$, Jose POZO 9 \\ 1 Research-Educational Institute of Optics and Biophotonics, Saratov State University (National Research University of Russia), \\ 410012 Saratov, Russia
}

2 Interdisciplinary Laboratory of Biophotonics, Tomsk State University (National Research University of Russia), 634050 Tomsk, Russia 3 Laboratory of Laser Diagnostics of Technical and Living Systems, Institute of Precision Mechanics and Control of RAS, 410028 Saratov, Russia 4 Institute of Electronics, Bulgarian Academy of Sciences, Sofia, Bulgaria

5 Faculty of Electronics, Telecommunication and Informatics, Gdańsk University of Technology, Poland

6 National Biophotonics and Imaging Platform Ireland, Chair of Applied Physics, School of Physics, National University of Ireland, Galway, Ireland

7 European Laboratory for Non Linear Spectroscopy (LENS) and University of Florence, Sesto Fiorentino, Italy

8 Leibniz Institute of Photonic Technology, Jena, Institute of Physical Chemistry \& Abbe Center of Photonics, Friedrich Schiller University, Jena, Germany

9 Technology and Innovation, EPIC - European Photonics Industry Consortium, Netherlands

(c) Higher Education Press and Springer-Verlag Berlin Heidelberg 2017

Biophotonics is an emerging multidisciplinary research area, embracing all light-based technologies applied to the life sciences and medicine [1-6]. The expression itself is the combination of the Greek syllables "bios" standing for life and "phos" standing for light. Photonics is the technical term for all methodologies and technologies utilizing photons over the whole spectrum from X-ray over the ultraviolet, visible and the infrared to the terahertz region, and its interaction with any matter. Beyond this definition, biophotonics is a scientific discipline of remarkable societal importance. As a part of photonics, it has proved to be an important enabling technology for accelerated progress in medicine and biotechnology. It can do so, because it originated at the interface of the most innovative academic disciplines of the last century, i.e., photonics, biotechnology and nanotechnologies. This field of research brings together scientists from different professions, such as physicists, biologists, pharmacists, medical doctors, etc. To work on common projects, they need to understand the ideas and the techniques of their counterparts.

Nobel prizes awarded in 2014 to Isamu Akasaki, Hiroshi Amano and Shuji Nakamura "for the invention of efficient blue light-emitting diodes which has enabled bright and energy-saving white light sources" and to Eric Betzig, Stefan W. Hell and William E. Moerner "for the development of super-resolved fluorescence microscopy" are related to biophotonics.

Nowadays, the necessity to gather information on a wide range of parameters appears to be crucial in many fields. It is especially important in medicine, health monitoring, and telemedicine. During recent years, biophotonics has gained popularity in those areas because of their unique advantages: relatively simple configuration, high resolution, potentially low cost and possibility of providing easy control and acquisition of measurement data.

With a global market estimated at about 430 billion euro in 2015, and at a CARG (Compound Annual Rate Growth) of $8.4 \%$ between 2016 and 2021 [7], the full potential of photonics is only now becoming clear. With predictions of double-digit growth in the future, biophotonics is one of the most promising of global markets. Valued at 32.37 billion euro in 2015, the biophotonics market is estimated to reach 86.24 billion euro by 2024 [8]. The biophotonics market covers a wide range of applications, ranging from tests and components (such as optical instruments, light sources for biophotonics, instrumentation and displays marketed specifically for bioscience applications), medical diagnostics (such as in vitro diagnostics assays and instruments, patient monitoring systems, imaging instrumentation and endoscopes using optics), medical therapeutics (such as surgical equipment and accessories, low-level laser therapy and radiation-based therapy), non-medical applications, biometric devices, and biosensing devices. 
The biophotonics marketplace has flourished because optical technologies enable imaging and treatment of human tissues and organs in real time at micron resolution and without the use of ionizing radiation. Only for one segment of the market, global sales of optical coherence tomography (OCT) systems have grown at an impressive compounded annual rate of about $45 \%$. The OCT system market was $\sim \$ 750$ million/year in 2015 and cumulative revenue over the past 25 years has surpassed $\sim \$ 5$ billion. The total federal government research investment over the past decade was $\sim \$ 500$ million (much of which was for the use of OCT in research not for developing fundamental OCT technology) and the total government tax receipts from OCT system companies and their direct employees was likely in excess of $\sim \$ 500$ million while taxes from indirect employment and component supply chain could far exceed $\sim 5 \mathrm{X}$ that number [9]. Rapid market growth will likely continue as clinical acceptance of biophotonics technologies becomes more widespread. Evidently, it has plenty of room to grow. At the same time, extensive development activity is leading to technical advances that are increasing biophotonics technologies utility and will further fuel market growth. Recent developments in the field of biophotonics are wide-ranging and include novel light sources that can extend the imaging range and treatment quality, innovative probe-delivery and light detection techniques, and the combination of optical techniques with other imaging modalities.

This Special Issue of Frontiers of Optoelectronics "Biophotonics in Europe" highlights achievements in biophotonics in this region. Papers collected, report on major developments and applications in the fields of biophotonics that have a significant impact. Prior to the analysis of the special issue content, it is important to present briefly major European activities in the field of biophotonics.

One of these major initiatives is Photonics4Life (P4L), which was launched in 2008 as the European Network of Excellence for Biophotonics. Supported by a four-year grant from the European Union over €4M, thirteen leading research institutes teamed up to provide a pan-European framework for research, clinical translation and education in biophotonics. In its four years of coordinated and dedicated action, P4L had yielded significant progress towards a coherent European platform for biophotonics. After the end of the funding period in 2012, the network included more than 100 research institutes as either a core partner, associated partner, or local cluster partner. At that point, the network presented itself as a diversified community of experts from academia, clinics, and industry. P4L had developed and successfully employed innovative tools to foster communication and collaboration between all these stakeholder groups and to facilitate appropriate education. The challenge was then to integrate the best part of these tools into a steady framework and give pan-European collaboration and networking in Biophotonics a long-term perspective. To that end, P4L was recently integrated into the worldwide community Biophotonics4Life which uses its newly-relaunched homepage (https://www.biophotonics.world/) to achieve impact and bundle forces in research dedicated to the benefit of human health. In collaboration with industry, Biophotonics4Life will further look into possibilities to support the translation of research into innovation on the way to marketable products and into the clinics for the future benefit of patients.

Photonics21 is the European Technology Platform (ETP) for optics and photonics industry. It creates a new entity for public-private partnership in photonics, funded by the European Commission's Horizon 2020 research and innovation plan. Work-group 3 "Life Science and Health" of Photonics21, chaired by Jürgen Popp aims to address an impending crisis for most European countries, the projected demographic changes will have drastic consequences for European citizens and their healthcare systems (www.photonics21.org/AboutPhotonics21/workgroup3.php). The group meets several times per year and largely sets the research agenda for Photonics for Health and Life Sciences in Europe. Some research initiatives involving photonics are set by the Nanomaterials group and the health group. The budget for photonics research supported by the Public Private Partnership of Photonics21 in the framework of the Horizon 2020 is €7BIn with a big portion of that is for Health and Life Sciences.

Different aspects of biophotonics are covered by European research groups and institutions. The integration tools, such as COST (European COoperation in Science and Technology) Actions and collaborative research projects and networks allow to increase the visibility of the scientific activities performed in different European countries, to share and exchange knowledge and to train young researchers. The COST scheme is an ultimate choice for development of such international networks, as it brings together COST-countries academia, industry and clinical end-users which would be virtually impossible through any other European funding mechanism. Examples, of such good practices are COST Actions, related to the biophotonics fundamental and applied research investigations, such as BM1205 "European network for skin cancer detection using laser imaging", MP1302 "NanoSpectroscopy" and the BMBS COST Action BM1401 Raman-based applications for clinical diagnostics (Raman4Clinics).

The very interesting and fruitful activity in the field of European biophotonics came to conclusion at March $18^{\text {th }}$, 2017 when COST Action European Network for Skin Cancer Detection using Laser Imaging (BM1205) was completed. The leader of the project and the Chair of the Action was Prof. Dragan Indjin from University of Leeds (UK). In the framework of this action, scientific centers from all over Europe tightened their cooperation around the topic of skin laser imaging. Participating were scientists (including three editors of this issue, Ekaterina Borisova, 
Malgorzata Jędrzejewska-Szczerska, and Valery V. Tuchin) from various fields, including clinicians, physicists and engineers who are involved in the design and development of optoelectronic devices that support skin laser imaging, such as laser sources and detection systems. During the period from 2013 to 2017, many short-term missions were financed for young and experienced scientists. Several meetings and schools related to the action topic were organized, providing invaluable opportunity for exchanging knowledge and experience, planning future research and cooperation.

Action BM1205 united partners from 28 countries (http://www.skin-laser-imaging.org/). It provided an interdisciplinary framework to enhance interaction activities within the field of optical biosensing, between worldclass academic groups, labs and system integrators from industry. It exploited novel laser sources and innovative feedback interferometry in specific biomedical applications. Recent developments in the design of solid-state laser sources at near-infrared, mid-infrared and terahertz frequencies, coupled with novel self-mixing approaches to signal detection and the integration of these into imaging platforms, provide a way forward in the design of the next generation of detection systems. All spectral range investigations, related to optical detection of neoplasia are exploited, covering ultraviolet, visible and infrared optical sensing and detection for diagnostic needs. Specifically extending the noninvasive interrogation of skin tissue into terahertz frequencies as well as new and promising imaging modalities were proposed. Action BM1205 exchanged knowledge, explored and compared technology platforms and performed clinical validation and evaluation of new devices which permitted detection of both the changes in skin lesions and disordered blood flow patterns and tissue perfusion typical for malignancy.

Another interesting and successful COST Action, directly involved biophotonics research is MP1302 "NanoSpectroscopy" (http://www.cost-nanospectroscopy.eu/). This Action consolidates European expertise on all aspects of UV/visible/NIR nanospectroscopy (modeling, experiment, nanostructures, materials, equipment, and applications) into one coherent Action including in the field of biomedical research and applications. The Action currently unites more than 200 participating research groups from 35 countries. Due to the fast development of nanosciences and in particular nanobiophotonics, there is an increasing demand for spectroscopic methods to investigate processes, objects, and material properties with unprecedented spatial and temporal resolution. The new insights are important for issues such as understanding life on the (sub-) cellular level, light-matter-interaction, or biomaterials engineering. The interdisciplinary approach of nanospectroscopy encompasses the fields of physics, biochemistry, biology, medicine, nanotechnology, and materials science and uses methods such as confocal and/or ultrafast Raman and fluorescence spectroscopy for the detection and spectral analysis of objects at the nanoscale, down to the single-molecule level. A third COST Action in the field of Biophotonics is Raman4Clinics (https://www. raman4clinics.eu/), which pools European expertise to progress in the field of novel, label-free and rapid technologies based on the wide variety of Raman spectroscopies for the clinical diagnostics of body fluids, bacteria, cells and tissues. The COST Action offers ample opportunity for international and interdisciplinary networking between scientists within biophotonics, chemometricians and physicians/clinicians. All participating research groups, at the moment 145 from 22 countries, are united in pursuing the goal of providing a major impetus in this vibrant field of research by aligning it to clinical requirements and application aspects (the unmet medical need) by means of COST as the best mechanism to progress the state-of-the-art. The Action constitutes an ideal platform for scientific communication, exchange, collaboration and for new research activities, combining the partners' expertise in technology, component, system and methodology development and medical application. As a result, novel technology portfolios for clinical diagnostics will emerge to the benefit of patients as well as to the economy.

Other initiatives for development of regional or European-level networks, working in the field of biophotonics and biomedical optics are also welcomed and supported by the researchers in different countries, with tools for development of common platform for investigations, training of young researchers, PhD and under graduated students, possibility to exchange a knowledge and research experience between the network members. One such regional network with increasing influence in the recent years united the countries from the Baltic region; The Baltic Sea Network "Nanotechnology meets (Bio)Photonics - NanoPhoto" (http://www.nanophoto.de/). The recently joined new members came from different countries around Europe and even third countries outside the EU member states, which make this network global initiative for association of investigators, working on the edge between biophotonics and nanotechnologies. This network's interests are in the development and testing of novel nanostructures such like bio-ceramic/bio-glasses, bio-silicon or semiconducting quantum dot based materials for cancer research (like labeling of living cell and cancer therapy). Researchers focus on the development of different nanostructures that most efficiently permit development of novel knowledge for cancer research. The partners involved in "NanoPhoto" are all from the areas of material science, physics, chemistry, biochemistry, biophotonics, biomedicine and are thus most ideally suited to develop the new materials and devices for biophotonics research and theranostic applications.

In its turn, the mission of The Ireland National Biophotonics and Imaging Platform (NBIPI) is to provide a structured research and training framework for Ireland's investment in advanced imaging applied to the Life Sciences and 
Medicine; to catalyze and support internationally competitive research grant applications; to develop and support Post-graduate Training Programmes underpinned by biophotonics and imaging; to bridge the Physical and Life Sciences interface; to provide a National Access for Core Facilities in Molecular, Cellular, Small Animal and Human Research Imaging; to provide the administrative and scientific infrastructure for Ireland's participation in large-scale international research programmes. This model has been adopted in several other European countries. The NBIPI is funded by the Irish Government's Programme for Research in Third Level Institutions, Cycle 4, Ireland's EU Structural Funds Programmes 2007-2013 and EU FP7. The platform consists of a consortium of imaging and biophotonics laboratories from across the Universities and Institutes of Technology in Ireland, 3 EU partners; CNRS Montpellier (France), the CNR Institute of Biostructure and Bioimaging, Naples (Italy) and The Nordic Imaging Network and 1 associated international partner; CECs (Chile). It has established many innovative initiatives, including the Career Enhancement and Mobility Programme (CEMP), BIGSS Summer School, National Access Charge policy approved by the national funding agency. Researchers can now include Access Charges for NBIPI equipment usage as a separate line item in the budget for all SFI or EU proposals. In its first four years, NBIPI has trained $>50 \mathrm{PhD}$ graduates directly funded by the platform since 2008 plus 60 postodctoral researchers. They are the next generation of multidisciplinary imaging scientists and have links with laboratories all over Europe and with Canadian, U.S. and Russian partners. It publishes $>100$ research articles per year in journals with an average impact factor $>5.1$.

The European Laboratory for Non-linear Spectroscopy (LENS), is a place where physicists, chemists and biologists work together every day, sharing instrumentation, experiences, research themes, scientific perspectives and ideas with the common aim of using laser light to investigate matter from different points of view and under different conditions. LENS is part of the European infrastructure Eurobiolmaging and of the Integrated Initiative of European Laser Research Infrastructures (Laserlab Europe). Research interests include atomic physics, photonics, biophysics, and chemistry, without forgetting advanced training of young researchers, thanks to e.g., a EU Marie Curie training program, through high quality $\mathrm{PhD}$ courses and a rich Post Doctoral fellowship program. Interdisciplinary is the keyword that best describes research activity at LENS: founded by a little group of scientists prevalently involved in atomic and molecular laser spectroscopy, in its 25 years of life LENS has grown developing and differentiating the research lines in new directions. All the research fields share the same fundamental methodology: the use of laser light to investigate matter.

The Biophysics Biophotonics Lab at LENS is involved in the European Flagship Human Brain Project (HBP), under the leading of Prof. Francesco Saverio Pavone, winner of an ERC Advanced Grant for the project BrainBIT: Alloptical brain-to-brain behaviour and information transfer. The Lab aims to develop innovative imaging methodologies for an increased understanding of biological structures and behaviours. In the HBP, novel implementations of lightsheet microscopy are applied by the LENS team to resolve neuronal anatomy in whole fixed brains with cellular resolution. The advantages of light-sheet illumination and confocal slit detection are combined to increase the image contrast in real time. Software for cell-counting and shape recognition in 3D brain images, based on deep learning strategies, are being developed. In living samples, real-time dynamics of brain rewiring are visualized through twophoton microscopy with the spatial resolution of single synaptic contacts. The plasticity of the injured brain is also dissected through cutting-edge optical methods that specifically ablate single neuronal processes. Finally, random access multi-photon microscopy in combination with novel fluorescence probes allows optical registration of action potentials across populations of neurons. The development and the application of these complementary optical methodologies provide fundamental insights in brain disease and represent a completely new approach for the investigation of the physiology of neuronal networks. LENS is applying the described approaches in both mouse (SP1) and human (SP2) subprojects in which it is involved.

The goal of the BrainBIT project is to establish, at the end of 2021, a novel paradigm of brain-to-brain communication based on direct full-optical recording and controlled stimulation of neuronal activity in different subjects. Exchange of information between different brains usually takes place through the interaction between bodies and the external environment. The project is developing optical technologies well beyond the state of the art for simultaneous neuronal "reading" and "writing" across large volumes and with high spatial and temporal resolution, targeted to the transfer of advantageous behaviour in physiological and pathological conditions.

One of the contributors to this special issue, Stefan Andersson-Engels has moved recently to the Tyndall National Institute in Ireland with a €5M start-up package from Science Foundation Ireland. Stefan is well known in the European Biophotonics Research community through his research at Lund and his co-chairing of the Ven Biophotonics Summer School which alternates with the Biophotonics and Imaging Graduate Summer School (BIGSS) in Ireland. Stefan in his review paper outlines some of his exciting and ambitious plans for deep tissue imaging which remains a huge challenge for the biophotonics community.

Europe is fortunate to have several summer schools dedicated to biophotonics. The Ven Biophotonics Summer 
School in Sweden (http://www.biop.dk/) and the Biophotonics and Imaging Graduate Summer School (BIGSS) in Ireland (http://www.nuigalway.ie/tomi/bigss2016.html) operate in alternate years and invite the leading professors from all over the world to teach the fundamentals of topic with which they are most familiar; Annual Summer School on Optics, Laser Physics \& Biophotonics as a part of Saratov Fall Meeting (this year is the $21^{\text {st }}$ meeting, September 25-29, 2017; http://sfm.eventry.org/2017/) and biannual "Photonics meets Biology" (this year is the $4^{\text {th }}$ meeting, September 19-22 2017, Tarragona, Spain; http://esperia.iesl.forth.gr/ mfarsari/accomodation.html). This gives a broad grounding across the hottest topics in biophotonics, creates life-long friendships with other students across the globe and direct contact with the leaders in the field. Through poster presentations, the students get feedback from very experienced professors, often saving them many months of work or directing them to unspotted potential in their work.

Two big European Symposia also should be mentioned. Each odd year sponsored by OSA and SPIE the European Conferences on Biomedical Optics (ECBO) (http://www.osa.org/en-us/meetings/topical_meetings/ european_conferences_on_biomedical_optics/) and each even year SPIE Photonics Europe with the Conference "Biophotonics: Photonic Solutions for Better Health Care" (Chairs: Jürgen Popp, Valery V. Tuchin, Francesco S. Pavone) (http://www.spie.org/EPE/conferencedetails/biophotonics-photonic-solutions?SSO = 1) bring together researchers, engineers and clinicians working with biomedical optics and biophotonics to solve problems in biology and medicine.

EPIC (European Photonics Industry Consortium) (http://www.epic-assoc.com/about-epic/) is the industry association that promotes the sustainable development of organizations working in the field of photonics in Europe. They foster a vibrant photonics ecosystem by maintaining a strong network and acting as a catalyst and facilitator for technological and commercial advancement. EPIC publishes market and technology reports, organizes technical workshops and B2B roundtables, supports EU funding proposals, advocacy and lobbying, education and training activities, standards and roadmaps, and pavilions at exhibitions. Europe needs to better exploit the innovation capacity of the bio-photonics SMEs, but also better organize how general photonics SMEs can be integrated in the health and life sciences sector. Moreover, Europe needs to utilize the innovation leverage potential of the innovation clusters and national platforms. To overcome that limitation, EPIC organizes a yearly Biophotonics workshop at a hospital location. In previous editions, this workshop has taken place at Maastricht MC (Netherlands), Erasmus MC (Netherlands), Berliner Klinik (Germany), Gustave Roussy (France). The 2017 edition will take place in at the Van Leeuwenhoek Hospital in Amsterdam. The purpose of this workshop is to bring together three different sets of stakeholders: technology developers, system integrators, and medical doctors, with the ultimate goal of enabling innovative upcoming products from SME reaching clinical trials.

The special issue is opened by a few reviews that highlight recent achievements in a particular field of biophotonics or in the multiple fields of the leading research groups in Europe. The review article by Jacqueline Gunther and Stefan Andersson-Engels presents a comprehensive overview of the recent studies on acousto-optical tomography (AOT) and its future biomedical applications. Authors outline the challenges that AOT still has as a technology to provide in vivo imaging, discuss recent achivements and future perspectives.

The review paper by Ronald Sroka et al. on research and development of laser techniques for translation into clinical application presents innovative clinical concepts and devices based on the Munich prolonged experience. Authors discuss fluorescence technologies for noninvasive diagnostics and disease monitoring, aiming to improve photodynamic therapy (PDT). Examples of thermal laser therapy of such pathologies as varicose veins and in lithotripsy are also given.

Janis Spigulis, in his paper entitled as In-vivo skin imaging prototypes "made in Latvia", reviews the portable in-vivo skin imaging prototypes developed at the Biophotonics Laboratory of Institute of Atomic Physics and Spectroscopy, University of Latvia. These prototypes provide distant mapping of skin chromophores, imaging of autofluorescence photobleaching rate for discrimination of skin tumor margins, and photoplethysmographic video-imaging.

The paper by Adam Cenian presents the state-of-art of laser technologies developed recently in Poland, including the development of laser techniques for medical diagnostics and therapy.

The next eight papers illustrate some of the exciting biophotonic studies recently done in Germany, Poland, Bulgaria, Russia, and Ukraine. In particular, Bernd Kampe et al. introduce support vector machine-based recursive feature elimination technique adapted to the multi-class setting for the analysis of Raman spectroscopic data received for clinically relevant microorganisms in urine samples, along with patient samples. X. Luís Deán-Ben et al. analyze and account for the effects of the speed of sound variations in 3-D hand-held optoacoustic tomographic acquisition geometries by using the proposed efficient GPU-based reconstruction framework. Jerzy Pluciński and Andrzej Frydrychowski, by using a Monte Carlo method applied to a multi-layer head tissue model, studied the influence of the pulse wave on the transmission of NIR radiation in outer head tissue layers caused by changes in tissue optical properties and the width of the subarachnoid layer. Excitation-emission matrices (EEMs) of 
autofluorescence for colorectal tumors were investigated by Ekaterina Borisova et al. as a tool for spectroscopic diagnostics of gastrointestinal neoplasia basing on detection of diagnostically-important chromophores, such as collagen, elastin, NADH, flavins, and porphyrins and their alterations during tumor development. Andrey V. Belikov et al. studied microperforation of human nail plate by radiation of Yb,Er:Glass (1540 nm) and Er:YLF (2810 nm) lasers aiming to quantify ablation effects. The technology developed is of great importance for drug delivery via nail plate. The paper by Sergey Savenkov et al. also describes studies on nails. Optical polarization measurements were proposed for tissue characterization in terms of depolarizing Mueller matrix decompositions. To prove the sensitivity of the technique, the Mueller matrices were measured for the nail samples before and after their irradiation by ionizing radiation. Dynamics of Pentoxifylline impact on the oxygen status of Pliss's lymph sarcoma in rats was investigated by Tatiana I. Kalganova et al. by using diffuse optical spectroscopy (DOS). Vasily A. Matkivsky et al. present a new method for detection and correction distortions of images received by spectral-domain OCT and caused by tissue chromatic dispersion. The method is robust and suitable for imaging of living tissues.

Overall, papers in this special issue cover some recent achievements in biophotonics. We hope the readers will find them interesting and inspiring. Evidently, this is only a small portion of very intensive research and development projects currently realized in Europe.

Finally, we would like to thank all authors for their great input in this issue and reviewers for their constructive comments.

\section{References}

1. Popp J, Tuchin V V, Chiou A, Heinemann S H, eds. Handbook of Biophotonics, vol. 1: Basics and Techniques; vol. 2: Photonics for Health Care; vol. 3: Photonics in Pharmaceutics, Bioanalysis and Environmental Research. Weinheim: WILEY-VCH Verlag GmbH \& Co. KGaA, 2011-2012, $2100 \mathrm{p}$

2. Tuchin V V. Dictionary of Biomedical Optics and Biophotonics. Bellingham, WA: SPIE Press, 2012, $576 \mathrm{p}$

3. Leahy M J, ed. Microcirculation Imaging. Weinheim: Wiley-VCH Verlag GmbH \& Co. KGaA, 2012

4. Pavone F S, Campagnola P J, eds. Second Harmonic Generation Imaging. Boca Raton: CRC Press, 2014

5. Tuan Vo-Dinh, ed. Biomedical Photonics Handbook. Boca Raton: CRC Press, 2015

6. Tuchin V V, ed. Handbook of Optical Biomedical Diagnostics, vol. 1 (PM262): Light-Tissue Interaction; vol. 2 (PM263): Methods. 2nd ed. Bellingham, WA: SPIE Press, 2016, $1552 \mathrm{p}$

7. http://www.marketsandmarkets.com/Market-Reports/photonics-market-88194993.html?gclid = CPrejKCEutICFYoQ0wodgroKbQ

8. http://www.grandviewresearch.com/press-release/global-biophotonics-market

9. Swanson E A. Optical coherence tomography: beyond better clinical care:OCT's economic impact. BioOptics World, 2016. http://www. bioopticsworld.com/articles/print/volume-9/issue-6/optical-coherence-tomography-beyond-better-clinical-care-oct-s-economic-impact.html

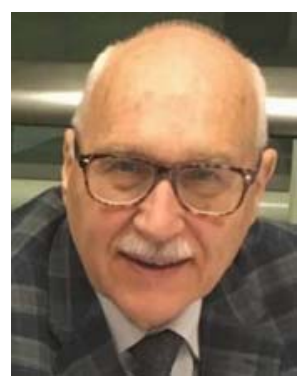

Valery V. Tuchin received a M.S. degree in Radio-Physics and Electronics (1966), a Ph.D. degree in Optics (1974), and a Doctor of Science in Laser Physics (1982) from Saratov State University. Currently he is a Professor and holds the Chair of Optics and Biophotonics of Saratov State University (National Research University of Russia). He is also a Director of the Research-Educational Institute of Optics and Biophotonics at SSU, Head of Laboratory on Laser Diagnostics of Technical and Living Systems, Institute of Precision Mechanics and Control, RAS, a supervisor of Interdisciplinary Laboratory on Biophotonics of Tomsk State University (National Research University of Russia), and Laboratory of Femtomedicine at ITMO University, Guest Professor of HUST (Wuhan) and Tianjin Universities of China, and Adjunct Professor of the Limerick University (Ireland) and National University of Ireland (Galway). His research interests include biophotonics, tissue optics, laser medicine, tissue optical clearing, and nanobiophotonics. He has published more than 700 papers (Web of Science), 27 monographs and text-books, and 58 book chapters. Prof. Tuchin is also a holder of more than 50 patents.

He is a fellow of SPIE and OSA, has been awarded Honored Science Worker of the Russia, Honored Professor of Saratov University, SPIE Educator Award, FiFiPro (Finland), Chime Bell Prize of Hubei Province (China), and Joseph W. Goodman Book Writing Award (OSA/SPIE). Prof. Tuchin is the recipient of the 2016 NanQiang Life Science Series Lectures Award of Xiamen University, China.

E-mail: tuchinvv@mail.ru 


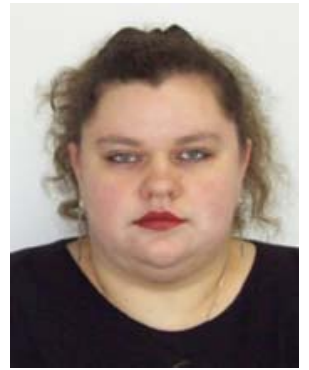

Ekaterina Borisova studied Medical Physics and Laser Physics in Physics Faculty of Sofia University, Bulgaria. The Ph.D. degree on Physics was received in Institute of Electronics, Bulgarian Academy of Sciences (IE-BAS) in 2005. From 2012 she became a Scientific Secretary of IE-BAS, from 2014 - Member of the Academic Council of BAS, and from March 2016 she was elected as a member of the Executive Council of BAS.

She is a co-author of six book chapters, more than 120 articles and conference full texts reports, as well as of five national and European patents in the field of biophotonics. Her recent investigations cover the field of molecular spectroscopy of biological samples, optical biopsy of human tissues and monitoring and detection of their pathological changes, as well as the problems of photodynamic medicine and its applications.

Dr. Borisova is a senior member of SPIE, and regular member of European Physics Society, European Society of Photobiology, European Platform of Photodynamic Medicine, and Union of Physicists in Bulgaria.

Her researches in the field of optical spectroscopy of human tissues for oncologic applications were honored with the BAS Prize for best young scientist in the field of Physics (2004), Prize "Pythagoras" of the Ministry of Education and Sciences for the Best Young Scientist in Bulgaria in 2012, as well as with an UNESCO/L'Oreal fellowship "For Women in Science" for Bulgaria in 2014.

E-mail: borisova@ie.bas.bg

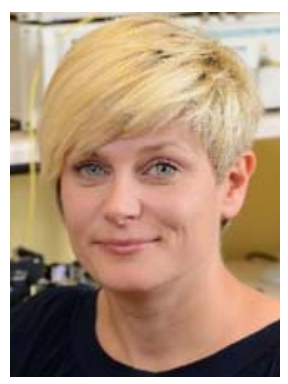

Malgorzata Jędrzejewska-Szczerska received her M.S. degree in Optoelectronics (1998), Ph.D. degree with honors in Electronics (2008) and a Doctor of Science in Electronics (2016) from the Gdańsk University of Technology. Currently, she is an assistant professor in the Department of Metrology and Optoelectronics. Her main research area is biophotonics and she focuses on the use of low-coherence interferometry, fiber-optic technology and application of optical measurements in biomedical applications.

She has authored more than 53 peer-reviewed papers, handbooks, monographs, tutorials, and book chapters. She is a member of SPIE and OSA. Furthermore, she is an advisor of the OSA Student Chapter at the Gdańsk University of Technology.

E-mail: mjedrzej@eti.pg.gda.pl

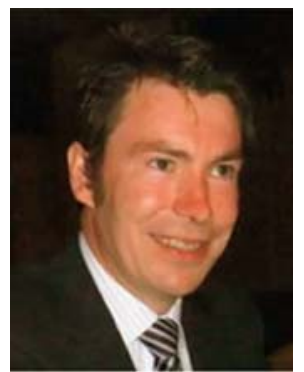

Martin J. Leahy completed a DPhil at the University of Oxford and he and a colleague established Oxford Optronix Ltd., where he was Director of R\&D. From 1995 he had various research and teaching posts at the University of Oxford. He then joined the Stokes Research Institute where he conducted industry led R\&D and later the Physics Department at the University of Limerick where led research groups in energy and biophotonics and lectured in physics. He has secured more than $€ 7 \mathrm{M}$ in external R\&D funding since 2003 . He is an adjunct professor at the Royal College of Surgeons, Fellow of the Institute of Physics, Fellow of the Royal Academy of Medicine in Ireland and Fellow of SPIE. He is a co-chair, executive organizing committee member, panel moderator for SPIE Photonics West BiOS in San Francisco and Editorial Board member of the Journal of Biomedical Optics, and host of the BioPIC European Bioimaging conference. Prof. Leahy was founding MD of Limerick West Windfarms Ltd. and Millstream Energy and PI on the research projects that spun out Biomass Heating Solutions Ltd. and Wheelsbridge AB. Since his return to full-time academia, he has played a leading role in graduate biophotonics education through NBIP, UL and the Biophotonics and Imaging Graduate Summer School. Since 2010 he has delivered more than 20 international invited lectures and published more than 40 ISI journal articles. He is currently Chair of Applied Physics at NUI Galway and was elected to Council of the Royal Microscopical Society in 2015.

E-mail: martin.leahy@nuigalway.ie

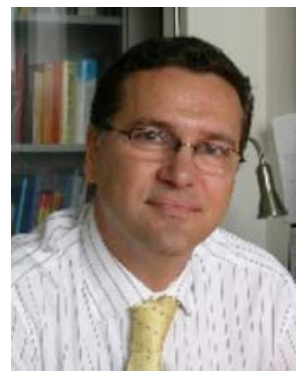

Francesco Saverio Pavone is Professor at the University of Florence, Department of Physics, and Director of the European Laboratory for Non-Linear Spectroscopy (LENS). He is leading a research group working in the field of biophotonics on single molecule biophysics, microscopy imaging-spectroscopy techniques, biomedical imaging, laser manipulation of bio-samples. In particular, he is developing new microscopy techniques for high resolution and high sensitivity imaging, and for laser manipulation purposes. These techniques have been applied both for single molecule biophysics, single cell imaging and optical manipulation. Tissue imaging is another research area developed, where non-linear optical techniques have been applied for skin and neural tissue imaging also in-vivo.

Pavone is author of many internationals papers and editor of international books. He has more than 100 invited talk and he is editors of international journals. He coordinates several European projects and he has organized several international congresses; he is also winner of a European Research Council Advanced Grant (2016-2021) and has an H-index equal to 37 (updating 31.03.2017).

E-mail: pavone@lens.unifi.it 


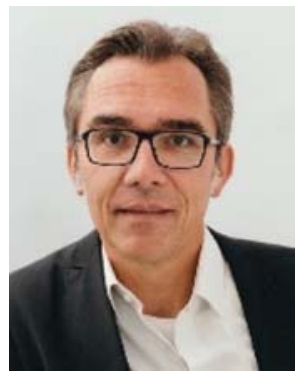

Jürgen Popp studied chemistry at the universities of Erlangen and Würzburg. After his Ph.D. in Chemistry, he joined Yale University for postdoctoral work. He subsequently returned to Würzburg University where he finished his habilitation in 2002. Since 2002, he holds a chair for Physical Chemistry at the Friedrich-Schiller University Jena. Since 2006, he is also the scientific director of the Leibniz Institute of Photonic Technology, Jena. His research interests are mainly concerned with biophotonics. In particular, his expertise in the development and application of innovative Raman techniques for biomedical diagnosis should be emphasized. He has published more than 630 journal papers and has been named as an inventor on 12 patents in the field of spectroscopic instrumentation. He is founding editor and Editor-in-Chief of the Journal of Biophotonics. In 2012, he received an honorary doctoral degree from Babeş-Bolyai University in Cluj-Napoca, Romania. Prof. Popp is the recipient of the 2013 Robert Kellner Lecture Award and the prestigious 2016 Pittsburgh Spectroscopy Award. In 2016, he was elected to the American Institute for Medical and Biological Engineering (AIMBE) College of Fellows.

E-mail: juergen.popp@ipht-jena.de

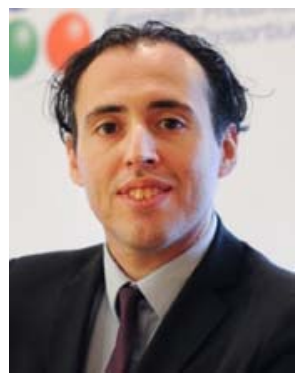

Jose Pozo is director of technology and innovation at EPIC (European Photonics Industry Consortium). As EPIC's CTO, he represents 300 leading corporate members active in the field of Photonics. He has 20 years' background in photonics technology, market knowledge, and a large network within the industrial and academic photonics landscape. Dr. Pozo holds a Ph.D. degree in Electrical Engineering from the University of Bristol, U.K., and a M.Sc. and B.Eng. degrees in Telecom Engineering from Spain/Belgium.

E-mail: jose.pozo@epic-assoc.com 